\title{
A Portable \& Disposable Ultra-Low Velocity Flow Sensor from Bioinspired Hair-Like Microstructures ${ }^{\dagger}$
}

\author{
Harish Devaraj 1, Rajnish Sharma 1, Enrico Haemmerle ${ }^{2}$ and Kean Aw 1,* \\ 1 Department of Mechanical Engineering, University of Auckland, Auckland 1010, New Zealand; \\ h.devaraj@auckland.ac.nz (H.D.); r.sharma@auckland.ac.nz (R.S.) \\ 2 School of Engineering, Computer and Mathematical Sciences, Auckland University of Technology, \\ Auckland 1010, New Zealand; enrico.haemmerle@aut.ac.nz \\ * Correspondence: k.aw@auckland.ac.nz; Tel.: +64-9-9239767 \\ + Presented at the Eurosensors 2018 Conference, Graz, Austria, 9-12 September 2018.
}

Published: 3 December 2018

\begin{abstract}
We present, for the first time, the design, development and testing of a portable ultra-low velocity flow sensor with a disposable architecture for use in medical applications. 3Dmicroprinting technique was used to fabricate high aspect ratio microscopic hair-like structures from conducting polymers, in particular, poly(3,4-ethylenedioxythiophene):polystyrene-sulfonate (PEDOT:PSS). These high aspect ratio micro-hairs are flexible and conductive that can respond to air flowing over them. A disposable and portable flow sensor with a modular design that allows tuning of measurement range was developed, for integration with an automated neonatal resuscitator to provide closed-loop feedback. The developed portable sensor architecture is capable of real-time indication of the air flow velocity range down to few millimeters/second.
\end{abstract}

Keywords: MEMS; velocity sensors; air flow sensor; conducting polymers; Micro switches; 3D microstructure printing

\section{Introduction}

Over 300,000 neonatal deaths/year have been reported worldwide due to birth asphyxia [1] and this number can greatly be reduced through timely administration of simple but effective neonatal resuscitation. Automation of this technique to ensure timely administration of the resuscitation has been recently realized [2]. This device is capable of administering a user-defined tidal volume to the lungs by means of high precision actuators accompanied by robust control electronics. This approach while proven to be effective, the lack of flow range feedback can potentially lead to hyperventilation or hypoventilation, both situations deemed to be extremely fatal. While numerous low-velocity flow sensors exist [3,4], they pose serious drawbacks when it comes to integration with automated medical resuscitators as they are complex to develop and more importantly the replacement of these flow sensors between uses to maintain hygiene standards of the medical procedure is not cost-effective. The expensive electronics to precisely measure the flow rate continuously and provide an analogue output of precise air flow rates is also superfluous for this particular medical application.

We present here, for the first time, a robust, portable and cost-effective disposable ultra-low velocity flow sensor with a digital response that indicates the flow rate range. Using a high-precision microstructure 3D printer, high aspect ratio micro-structures were fabricated from conducting polymers, PEDOT:PSS in particular. These high aspect ratio micro-hairs are highly flexible and conductive at the same time and can bend about their anchor point under the application of external force (drag force) experienced under low velocity air flows overthem. 


\section{Materials and Methods}

\subsection{Sensing Micro-Hair Fabricaiton}

PEDOT:PSS dispersion (1.3 wt $\%$ in $\mathrm{H} 2 \mathrm{O}$ ) is dissolved in dimethyl sulfoxide (DMSO) to improve the adhesion of PEDOT:PSS to the gold substrate while increasing the conductivity of the dispersion. A custom built high precision 3D-microstructure printer capable of creating nanometer scale 3D features was used to fabricate the micro-hairs for the flow sensor [5]. Figure 1 shows the time-lapse microscope image of the micro-hair printing process and the SEM image of a sample array of microhairs created using this process. The response of the micro-hairs to air flow solely depending on their stiffness, an micro-hair aspect ratio of $181.2 \pm 15$ ( $1 \mathrm{~mm}$ high, $5.5 \pm 5 \mu \mathrm{m}$ in diameter) were identified to be ideal with enough sensitivity to low velocity air flows and stiffness to ensure return to their initial state when the external stimulus is removed. Under an applied air flow the micro-hairs bend and make contact with a contact terminal placed mutually perpendicular to the micro-hairs axis and the flow direction.

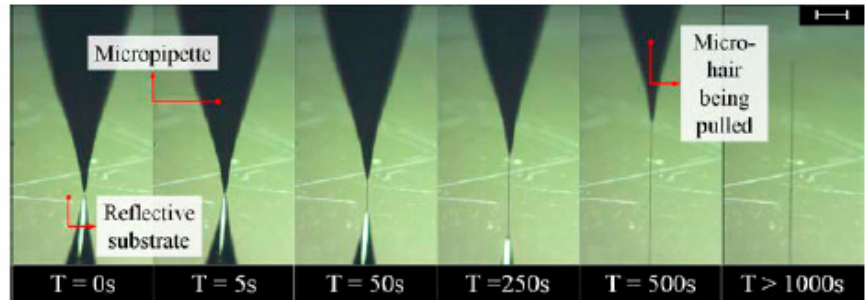

(a)

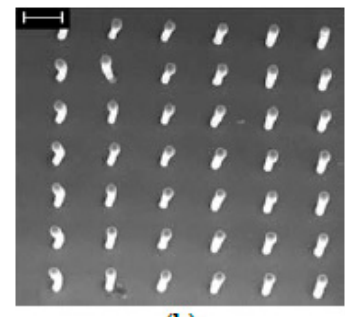

(b)

Figure 1. Time lapse sequence showing fabrication process of micro-hairs (scale bar $-150 \mu \mathrm{m})$; (b) SEM image of a representative micro-hair array at $10^{\circ}$ stage tilt (scale bar $\left.-20 \mu \mathrm{m}\right)$.

\subsection{Disposable Micro-Hairsensing Element}

Neonatal resuscitation being the intended target application demands sensors with high levels of hygiene capable of measuring flows between $1-8 \mathrm{~mL} / \mathrm{s}$. To allow replacement of the sensing element between each use to maintain the hygiene standards, $5 \mathrm{~mm} \times 5 \mathrm{~mm}$ two sided PCB were designed (Figure 2a). This PCB has 8 gold plated electrodes (pitch of $0.5 \mathrm{~mm}$ ) on either side for connecting to the sensor electronics via a $0.5 \mathrm{~mm}$ 8-pin FFC connecting cable and allow printing of the sensing micro-hairs on the other side. Single micro-hair was precisely printed on each trace at specific distances (Table 1) [6] relative to each other to allow sensing of flows in the range $1-8 \mathrm{~mL} / \mathrm{s}$ through a $15 \mathrm{~mm}$ inlet. This PCB is slotted into the flow channel's recess as shown in Figure $2 \mathrm{~b}$. The flow channel itself is casted from PDMS with the recess to hold the sensing element PCB ensuring the top surface of the sensing element PCB sits flush with the flow channel's walls.

Table 1. Inlet flow velocities and the required distance between the micro-hair and contact terminal for contact to be established at these velocities and the corresponding sensor output.

\begin{tabular}{cccc}
\hline $\begin{array}{c}\text { Air Flow Velocity } \\
\text { At Inlet }(\mathbf{m m} / \mathbf{s})\end{array}$ & $\begin{array}{c}\text { Theoretical Distance } \\
\text { between Micro-Hair and } \\
\text { Contact Terminal }(\boldsymbol{\mu m})\end{array}$ & $\begin{array}{c}\text { Sensor Output Signal } \\
\text { on the LED Indicator } \\
(\mathbf{1}-\text { ON/0-OFF) }\end{array}$ & $\begin{array}{c}\text { Flow Range Read } \\
\text { Out } \\
(\mathbf{m i n}-\mathbf{m a x}) \\
(\mathbf{m L} / \mathbf{s})\end{array}$ \\
\hline & & 00000000 & $0-1$ \\
5.7 & 13 & 10000000 & $1-2$ \\
11.3 & 30 & 11000000 & $2-3$ \\
16.9 & 50 & 11100000 & $3-4$ \\
22.6 & 67 & 11110000 & $4-5$ \\
28.3 & 88 & 11111000 & $6-7$ \\
33.9 & 110 & 11111100 & $7-8$ \\
39.6 & 132 & 11111110 & $8-9$ \\
45.3 & 155 & 11111111 & $>9$ \\
\hline
\end{tabular}




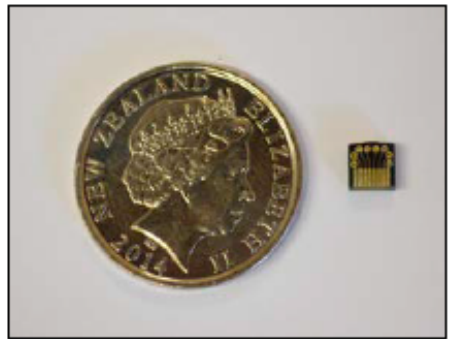

(a)

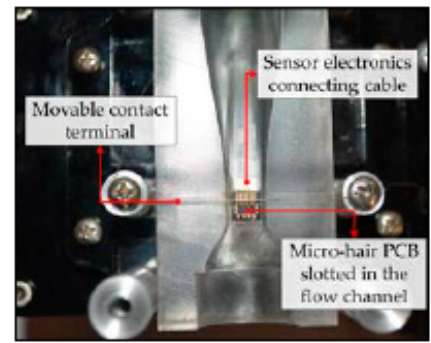

(b)

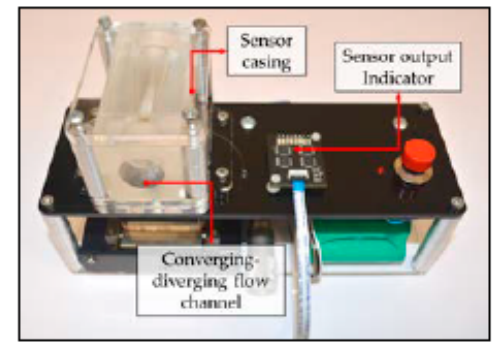

(c)

Figure 2. (a) The disposable micro-hair PCB next to a 50C coin (b) micro-hair PCB slotted in the throat of the converging-diverging flow channel with the FFC connecting to the sensor electronics visible beneath the translucent PDMS channel (c) The flow channel in the portable module with the power source and sensor electronics.

\section{Results}

The replacement of sensing element demands manual handling of the sensing element PCB while its placement in the recess and the micro-hair's dimension of $\phi 5.5 \pm 0.5 \times 1 \mathrm{~mm}$ only allow sensing of low-velocity flows $(0.66-0.97 \mathrm{~m} / \mathrm{s})$. A gradually converging and diverging flow channel amplifies the air-flow velocity enabling measurement of ultra-low velocity air flows with stiffer micro-hairs. The two halves of the soft flow channel ensures leakless flow through the channel retaining the flow regime and the inlet and the outlet pressure. The channel is fastened down within a Perspex enclosure mounted above a stationary platform. This platform also houses a precision micrometric stage which holds the contact terminal in place suspended above the sensing element PCB. The movement of this stage moves the contact terminal allowing replacement of the sensing element PCB without damaging the micro-hairs during the assembly process (Figure 3a).

After positioning the sensing element $\mathrm{PCB}$, the contact terminal is then moved towards the micro-hairs while the sensor is powered on. As the contact terminal makes contact with the first micro-hair, the current flow through this micro-hair is picked up by the sensor electronics and turns on the indicator LED. From this point, the contact terminal is moved away in the opposite direction by a distance of $13 \mu \mathrm{m}$. This distance is estimated based on the target flow rates [6] so as to initiate contact of the first micro-hair only above $1 \mathrm{~mL} / \mathrm{s}$ flow through the $15 \mathrm{~mm}$ inlet.

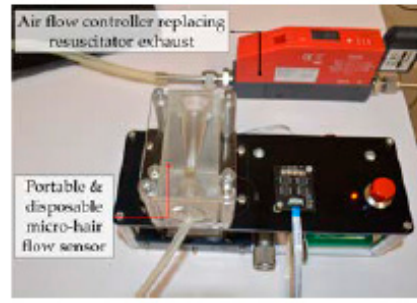

(a)

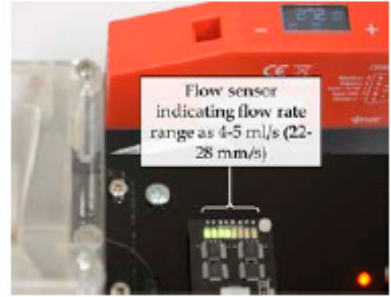

(b)

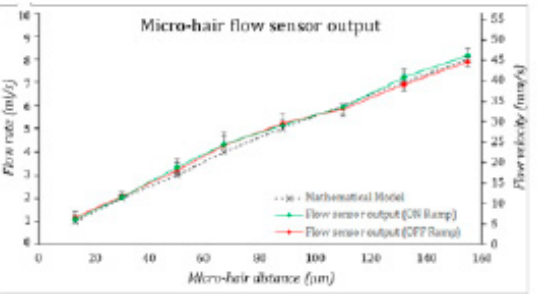

(c)

Figure 3. (a) The portable flow sensor with integrated electronics connected to a Vögtlin gas flow meter. (b) The flow velocity range being indicated through LEDs as each micro-hair closes and establishes contact over particular flow velocities (display on the gas flow meter is in $\mathrm{ml} / \mathrm{min}$ ). (c) Sensor output showing high repeatability with low hysteresis across multiple flow cycles.

The flow sensor's performance is validated by connecting the device to a Vögtlin mass flow controller. The controller is connected to precisely allow a specific flow rate analogous to the automated resuscitator as shown in Figure 3a. The outlet flow from this controller is connected to the micro-hair flow sensor's inlet. The flow is ramped up and down across the entire flow range and the flow rates at which each micro-hair makes contact with the contact terminal is recorded at the instant individual LEDs turn on (Figure 3b). Figure 3c shows the sensor's response to the air flow. 
It was observed that the sensor output, when compared to the sensor's response estimated from the mathematical model [6] had a maximum deviation of $13 \%\left(0.5 \mathrm{ml} . \mathrm{s}^{-1}\right)$. This is primarily attributed to the error introduced in the straightness of the contact terminal during the manual assembly process. Furthermore, the mathematical tool developed based on Lattice-Boltzmann method and large deflection models to estimate the micro-hair's response does not take into account factors such as the surface roughness of the micro-hair, parasitic drag, oscillations induced due to neighboring micro-hairs and the straightness of the contact terminal. Nevertheless, the mathematical tool was invaluable in predetermining the micro-hair distances from the contact terminal, reducing the need for trial and error approach to design the flow sensor for measuring particular flow rates.

\section{Conclusions}

The high aspect ratio induced sagging of the cantilever limits the shelf life to each sensing element to 6 days and this can be further increased by storing them suspended upside down. In-situ printing of high aspect-ratio micro hairs is an alternative method to ensure accurate sensor response. The sensing element materials i.e., the PCB and PEDOT:PSS for fabricating the micro-hairs are cheap enough to allow replacement of the sensing element after each use The flow sensor's measurement range can also be tuned by changing the position of the contact terminal, detailed analysis of which is beyond the scope of this research. This biocompatible, cost-effective ( $0.20 \mathrm{NZD} /$ sensing element), portable and disposable flow sensor for measuring ultra-low velocity flows suitable for medical use demonstrates biocompatibility of PEDOT:PSS in medical applications, further studies from a clinical point of view is to be carried out.

Author Contributions: H.D. and K.A. conceived and designed the experiments; H.D. performed the experiments, collected and analyzed the data; R.S. contributed to the mathematical models used; E.H. provided input to optimize the device to meet the operating conditions of the target application; H.D. and K.A. wrote the paper.

Acknowledgments: The authors would like to thank University of Auckland for providing financial support to undertake this research.

Conflicts of Interest: The authors declare no conflict of interest.

\section{References}

1. WHO. World Health Statistics, 2015; WHO: Geneva, Switzerland, 2015; pp. 15-16.

2. Solevåg, A.L.; Haemmerle, E.; van Os, S.; Bach, K.P.; Cheung, P.-Y.; Schmölzer, G.M. A novel prototype neonatal resuscitator that controls tidal volume and ventilation rate: A comparative study of mask ventilation in a new-born manikin. Front. Pediatr. 2016, 4,129.

3. Maschmann, M.R.; Ehlert, G.J.; Dickinson, B.T.; Phillips, D.M.; Ray, C.W.; Reich, G.W.; Baur, J.W. Bioinspired carbon nanotube fuzzy fiber hair sensor for air-flow detection. Adv. Mater. 2014, 26, 3230-3234.

4. Schena, E.; Massaroni, C.; Saccomandi, P.; Cecchini, S. Flow measurement in mechanical ventilation: A review. Med. Eng. Phys. 2015, 37, 257-264.

5. Devaraj, H.; Travas-Sejdic, J.; Sharma, R.; Aydemir, N.; Williams, D.; Haemmerle, E.; Aw, K.C. Bioinspired flow sensor from printed PEDOT:PSS micro-hairs. Bioinspir. Biomim. 2015, 10,016017.

6. Devaraj, H.; Aw, K.C.; Haemmerle, E.; Sharma, R. Fluid-structure interaction of high aspect-ratio hair-like micro-structures through dimensional transformation using lattice boltzmann method. Int. J. Appl. Mech. 2016, 8, 1650095 .

(C) 2018 by the authors. Licensee MDPI, Basel, Switzerland. This article is an open access article distributed under the terms and conditions of the Creative Commons Attribution (CC BY) license (http://creativecommons.org/licenses/by/4.0/). 